\title{
Existence of Positive Periodic Solutions for a Time-Delay Biological Model
}

\author{
Binbin Wang, Hailiang Zhang* \\ Department of Mathematics, Zhejiang Ocean University, Zhoushan, China \\ Email: "hlzhang88wy@163.com
}

Received 31 March 2016; accepted 12 July 2016; published 15 July 2016

\begin{abstract}
Based on the classic Lotlk-Volterra cooperation model, we establish a time-delay model of which a species cannot survive independently. By continuation theorem, we discuss existence of positive periodic solutions of the model.
\end{abstract}

\section{Keywords}

\section{Biological Model, Existence, Periodic Solution, Time Delay, Independent Survival}

\section{Introduction}

The plants can survive independently and insect pollination can improve the growth rate of plants in [1] and [2]. According to this phenomenon, based on the classical Lotka-Volterra model, we establish a model of two populations of Lotka-Volterra which cannot survive independently, finally he analyzes the stability of the model.

There is still less research work of the model which cannot exist independently. The existing researches basically are the autonomous models (see [3] and [4]). In this paper, we establish a Lotka-Volterra model with time delay which a species cannot survive independently. The main aim is to discuss existence of periodic positive solution for the model.

Suppose that there are two plant populations (A and B) living in their natural environment, which are free from other interference factor. Let $y_{1}(t)$ and $y_{2}(t)$ are the population density of plant A and plant B, $a_{1}, a_{2}, b_{1}, b_{2}$ are continuous functions with periodic $\omega>0$, and $\int_{0}^{\omega} r_{i}(t) d t>0(i=1,2)$. The constants

$a_{1}, a_{2}, b_{1}, b_{2}$ are stimulations of living environment. By the thought of [1]-[4], we could have got the following Lotka-Volterra model with time delay which a species cannot survive independently.

$$
\left\{\begin{array}{l}
\frac{d y_{1}(t)}{d t}=y_{1}(t)\left[-r_{1}(t)-a_{1}(t) y_{1}(t-\tau(t))+b_{1}(t) y_{2}(t-\sigma(t))\right], \\
\frac{d y_{2}(t)}{d t}=y 2(t)\left[r_{2}(t)+a_{2}(t) y_{1}(t-\tau(t))-b_{2}(t) y_{2}(t-\sigma(t))\right] .
\end{array}\right.
$$

The main aim of the paper is to discuss existence of periodic positive solution for the model.

${ }^{*}$ Corresponding author. 


\section{Lemma 1 and Lemma 2}

Assume $\mathrm{X}$ and $\mathrm{Z}$ are normed vector space, $L: D o m L \subset X \rightarrow Z$ and $N: X \rightarrow Z$ are linear mappings. If $\mathrm{L}$ is Fredholm mapping which Zero is index, and there are continuous projection $P: X \rightarrow X$ and $Q: Z \rightarrow Z$, such that $\operatorname{Im} P=\operatorname{Ker} L$ and $\operatorname{Im} \operatorname{Ker} Q=\operatorname{Im}(I-Q)$, we can get that $L \mid \operatorname{Dom} L \cap \operatorname{Ker} P:(I-P) X \rightarrow \operatorname{Im} L$ is reversible. If Inverse mapping $K_{p}(I-Q) N: \bar{\nabla} \rightarrow X$ is tight, we call $\mathrm{N}$ is tight on $\bar{\nabla}$.

Lemma 1 (Continuation theorem) [5] Let $L$ be the mapping of Fredholm with zero index, collection $\mathrm{N}$ is tight on collection $\bar{\nabla}$. Suppose the following: for any $\lambda \in(0,1)$, the solution $x \notin \partial \nabla$ of equation $L x=\lambda N x$; for any $x \in \partial \nabla \cap \operatorname{Ker} L, Q N x \neq 0$ and $\operatorname{deg}\{J Q N, \nabla \cap \operatorname{KerL}, 0\} \neq 0$. Then, there is at least a solution for $L x=N x$ on $\operatorname{DomL} \cap \bar{\nabla}$.

Lemma $2 R_{+}^{2}=\{(x, y) \mid x>0, y>0\}$ is positive invariant set of model (1).

Proof: By formula (2), we have

$$
\left\{\begin{array}{l}
y_{1}(s)=y_{1}(0) \exp \left\{\int_{0}^{s}\left[-r_{1}(t)-a_{1}(t) y_{1}(t-\tau(t))+b_{1}(t) y_{2}(t-\sigma(t))\right] d t\right\}, \\
y_{2}(s)=y_{2}(0) \exp \left\{\int_{0}^{s}\left[-r_{2}(t)-a_{2}(t) y_{1}(t-\tau(t))+b_{1}(t) y_{2}(t-\sigma(t))\right] d t\right\} .
\end{array}\right.
$$

Since formula (2) is always true for $t \geq 0$, lemma 2 is proved.

For the convenience of discuss, we give following notations.

$$
\begin{gathered}
\bar{g}=\frac{1}{\omega} \int_{0}^{\omega} g(t) d t, \quad g^{M}=\max _{t \in[0, \omega]} g(t), \quad g^{m}=\min _{t \in[0, \omega]} g(t) \\
a_{1}^{m}=\min _{0 \leq t \leq \omega}\left\{a_{1}(t)\right\}, \quad a_{2}^{M}=\max _{0 \leq t \leq \omega}\left\{a_{2}(t)\right\}, \quad b_{1}^{M}=\max _{0 \leq t \leq \omega}\left\{b_{1}(t)\right\}, \quad b_{2}^{m}=\min _{0 \leq t \leq \omega}\left\{b_{2}(t)\right\} .
\end{gathered}
$$

\section{Existence of Periodic Solutions}

In order to apply Continuation theorem to system (3), we define

$$
X=Z=\left\{x(t)=\left(x_{1}(t), x_{2}(t)\right)^{T} \in C\left(R, R^{2}\right), x(t+\omega)=x(t)\right\},
$$

and

$$
\|x\|=\left\|\left(x_{1}(t), x_{2}(t)\right)^{T}\right\|=\max _{t \in[0, \omega]}\left|x_{1}(t)\right|+\max _{t \in[0, \omega]}\left|x_{2}(t)\right|\|x\|=\left\|\left(x_{1}(t), x_{2}(t)\right)^{T}\right\|=\max _{t \in[0, \omega]}\left|x_{1}(t)\right|+\max _{t \in[0, \omega]}\left|x_{2}(t)\right|,
$$

then $X, Z$ is Banach space under the norm $\|\bullet\|$ (see [6] and [7]).

Let $y_{1}(t)=\exp \left\{x_{1}(t)\right\}, y_{2}(t)=\exp \left\{x_{2}(t)\right\}$, the Equation (1) can be turns into

$$
\begin{aligned}
& \frac{d x_{1}(t)}{d t}=-r_{1}(t)-a_{1}(t) \exp \left\{x_{1}(t-\tau(t))\right\}+b_{1}(t) \exp \left\{x_{2}(t-\sigma(t))\right\}, \\
& \frac{d x_{2}(t)}{d t}=r_{2}(t)+a_{1}(t) \exp \left\{x_{1}(t-\tau(t))\right\}-b_{1}(t) \exp \left\{x_{2}(t-\sigma(t))\right\} .
\end{aligned}
$$

Since $u(t)=\left(x_{1}(t), x_{2}(t)\right)$ is periodic, we know that

$$
\Phi_{1}(u, t)=-r_{1}(t)-a_{1}(t) \exp \left\{x_{1}(t-\tau(t))\right\}+b_{1}(t) \exp \left\{x_{2}(t-\sigma(t))\right\}
$$

and

$$
\Phi_{2}(u, t)=r_{2}(t)+a_{1}(t) \exp \left\{x_{1}(t-\tau(t))\right\}-b_{1}(t) \exp \left\{x_{2}(t-\sigma(t))\right\}
$$

are continuous function with the periodicity $\omega$.

Let $N x=\left(\begin{array}{l}\Phi_{1}(u, t) \\ \Phi_{2}(u, t)\end{array}\right), \quad L x=\dot{x}=\frac{d x(t)}{d t}, \quad P x=\frac{1}{\omega} \int_{0}^{\omega} x(t) d t$, for $x \in X, Q z=\frac{1}{\omega} \int_{0}^{\omega} z(t) d t$, for $z \in Z Z \in Z$, then $\operatorname{Ker} L=R^{2}, \operatorname{Im} L=\left\{z \in Z: \int_{0}^{\omega} z(t) d t=0\right\}$ is closed set in set $\mathbf{Z}$,

$\operatorname{dim} \operatorname{Ker} L=2=\operatorname{codim} \operatorname{Im} L$, and $P, Q P$ and $Q$ is the continuous projection, such that $\operatorname{Im} P=\operatorname{KerL}$, $\operatorname{Ker} Q=\operatorname{Im} L=\operatorname{Im}(I-Q)$. Thus there is the inverse mapping of $L \quad K p: \operatorname{Im} L \rightarrow \operatorname{KerP} \cap \operatorname{DomL}$ and

$K p(z)=\int_{0}^{t} z(s) d s-\frac{1}{\omega} \int_{0}^{\omega} \int_{0}^{t} z(s) d s d t$. 
So that we get

$$
\begin{gathered}
Q N x=\left(\begin{array}{l}
\frac{1}{\omega} \int_{0}^{\omega} \Phi_{1}(u, s) d s \\
\frac{1}{\omega} \int_{0}^{\omega} \Phi_{2}(u, s) d s
\end{array}\right), \\
K p(I-Q) N x=\left(\begin{array}{l}
\int_{0}^{t} \Phi_{1}(u, s) d s \\
\int_{0}^{t} \Phi_{2}(u, s) d s
\end{array}\right)-\left(\begin{array}{l}
\frac{1}{\omega} \int_{0}^{\omega} \int_{0}^{t} \Phi_{1}(u, s) d s d t \\
\frac{1}{\omega} \int_{0}^{\omega} \int_{0}^{t} \Phi_{2}(u, s) d s d t
\end{array}\right)-\left(\begin{array}{l}
\left(\frac{t}{\omega}-0.5\right) \int_{0}^{\omega} \Phi_{1}(u, s) d s \\
\left(\frac{t}{\omega}-0.5\right) \int_{0}^{\omega} \Phi_{2}(u, s) d s
\end{array}\right) .
\end{gathered}
$$

It is obvious that $Q N$ and $K p(I-Q) N$ is continuous.

We assume that $\nabla$ is bounded open set. It is obvious that $Q N(\bar{\nabla})$ is bounded. We have that $\overline{K p(I-Q) N(\bar{\nabla})}$ is compact set by Arzela-Ascoli theorem, so we get $N$ is $L$-tight on $\bar{\nabla}$.

The corresponding operator equation is $L x=\lambda N x$ with $\lambda \in(0,1)$, we have the following formula

$$
\begin{gathered}
\frac{d x_{1}(t)}{d t}=\lambda\left\{-r_{1}(t)-a_{1}(t) \exp \left\{x_{1}(t-\tau(t))\right\}+b_{1}(t) \exp \left\{x_{2}(t-\sigma(t))\right\}\right\} \\
\frac{d x_{2}(t)}{d t}=\lambda\left\{r_{2}(t)+a_{1}(t) \exp \left\{x_{1}(t-\tau(t))\right\}-b_{1}(t) \exp \left\{x_{2}(t-\sigma(t))\right\}\right\} .
\end{gathered}
$$

We assume that $x=x(t) \in X$ is the solution for system (4) with $\lambda \in(0,1)$, by integral we get the following formula (5)

$$
\begin{aligned}
& \int_{0}^{\omega}-r_{1}(t)-a_{1}(t) \exp \left\{x_{1}(t-\tau(t))\right\}+b_{1}(t) \exp \left\{x_{2}(t-\sigma(t))\right\} d t=0, \\
& \int_{0}^{\omega} r_{2}(t)+a_{2}(t) \exp \left\{x_{1}(t-\tau(t))\right\}-b_{2}(t) \exp \left\{x_{2}(t-\sigma(t))\right\} d t=0 .
\end{aligned}
$$

To move term from one side of an algebraic equation to the other side, reversing its sign to maintain equality, we get the following

$$
\begin{aligned}
& \int_{0}^{\omega}-r_{1}(t)+b_{1}(t) \exp \left\{x_{2}(t-\sigma(t))\right\} d t=\int_{0}^{\omega} a_{1}(t) \exp \left\{x_{1}(t-\tau(t))\right\} d t . \\
& \int_{0}^{\omega} r_{2}(t)+a_{2}(t) \exp \left\{x_{1}(t-\tau(t))\right\} d t=\int_{0}^{\omega} b_{2}(t) \exp \left\{x_{2}(t-\sigma(t))\right\} d t .
\end{aligned}
$$

From formula (5), formula (6) and formula (7), we have

$$
\begin{aligned}
& \int_{0}^{\omega}\left|\dot{x_{1}}(t)\right| d t=\lambda \int_{0}^{\omega}-r_{1}(t)-a_{1}(t) \exp \left\{x_{1}(t-\tau(t))\right\}+b_{1}(t) \exp \left\{x_{2}(t-\sigma(t))\right\} d t \\
& \leq \int_{0}^{\omega}-r_{1}(t)+b_{1}(t) \exp \left\{x_{2}(t-\sigma(t))\right\} d t+\int_{0}^{\omega} a_{1}(t) \exp \left\{x_{1}(t-\tau(t))\right\} d t \\
& \leq 2 \int_{0}^{\omega} a_{1}(t) \exp \left\{x_{1}(t-\tau(t))\right\} d t .
\end{aligned}
$$

and

$$
\begin{aligned}
& \int_{0}^{\omega}\left|\dot{x}_{2}(t)\right| d t=\lambda \int_{0}^{\omega} r_{1}(t)+a_{2}(t) \exp \left\{x_{1}(t-\tau(t))\right\}-b_{2}(t) \exp \left\{x_{2}(t-\sigma(t))\right\} d t \\
& \leq \int_{0}^{\omega} r_{2}(t)+a_{2}(t) \exp \left\{x_{1}(t-\tau(t))\right\} d t+\int_{0}^{\omega} b_{2}(t) \exp \left\{x_{2}(t-\sigma(t))\right\} d t \\
& \leq 2 \int_{0}^{\omega} b_{2}(t) \exp \left\{x_{2}(t-\sigma(t))\right\} d t .
\end{aligned}
$$

From formula (8) and formula (9) we can get

$$
\begin{aligned}
& b_{1}^{m} \int_{0}^{\omega} \exp \left\{x_{1}(t-\tau(t))\right\} d t \leq-\bar{r}_{1} \omega+b_{1}^{M} \int_{0}^{\omega} \exp \left\{x_{2}(t-\sigma(t))\right\} d t . \\
& b_{2}^{m} \int_{0}^{\omega} \exp \left\{x_{2}(t-\sigma(t))\right\} d t \leq \overline{r_{2}} \omega+a_{2}^{M} \int_{0}^{\omega} \exp \left\{x_{1}(t-\tau(t))\right\} d t .
\end{aligned}
$$


From formula (10) and formula (11) we get

$$
\begin{aligned}
& b_{2}^{m} \int_{0}^{\omega} \exp \left\{x_{2}(t-\sigma(t))\right\} d t \leq \overline{r_{2}} \omega+a_{2}^{M} \int_{0}^{\omega} \exp \left\{x_{1}(t-\tau(t))\right\} d t \\
& \leq \bar{r}_{2} \omega+\frac{a_{2}^{M}}{a_{1}^{m}}\left(-\bar{r}_{1} \omega+b_{1}^{M} \int_{0}^{\omega} \exp \left\{x_{2}(t-\sigma(t))\right\} d t\right) .
\end{aligned}
$$

So that

$$
\int_{0}^{\omega} \exp \left\{x_{2}(t-\sigma(t))\right\} d t \leq \frac{\left(\overline{r_{2}} a_{1}^{m}-\overline{r_{1}} a_{2}^{M}\right) \omega}{a_{1}^{m} b_{2}^{m}+a_{2}^{M} b_{1}^{M}} \leq \frac{\left(\overline{r_{2}} a_{1}^{m}+\bar{r}_{1} a_{2}^{M}\right) \omega}{a_{1}^{m} b_{2}^{m}+a_{2}^{M} b_{1}^{M}} .
$$

Similarly, we have

$$
\int_{0}^{\omega} \exp \left\{x_{1}(t-\tau(t))\right\} d t \leq \frac{\left(\overline{r_{1}} b_{2}^{m}-\overline{r_{1}} b_{2}^{M}\right) \omega}{a_{1}^{m} b_{2}^{m}-a_{2}^{M} b_{1}^{M}} \leq \frac{\left(\overline{r_{1}} b_{2}^{m}+\overline{r_{1}} b_{2}^{M}\right) \omega}{a_{1}^{m} b_{2}^{m}-a_{2}^{M} b_{1}^{M}} .
$$

Using formula (7) we get

$$
b_{2}^{M} \int_{0}^{\omega} \exp \left\{x_{2}(t-\sigma(t))\right\} d t \geq \overline{r_{2}} \omega
$$

Thus

$$
\frac{r_{2} \omega}{b_{1}^{M}} \leq \int_{0}^{\omega} \exp \left\{x_{2}(t-\sigma(t))\right\} d t \leq \frac{\left(\overline{r_{2}} a_{1}^{m}+\overline{r_{1}} a_{2}^{M}\right) \omega}{a_{1}^{m} b_{2}^{m}+a_{2}^{M} b_{1}^{M}} .
$$

Similarly, we have

$$
-\frac{r_{1} \omega}{a_{1}^{M}} \leq \int_{0}^{\omega} \exp \left\{x_{1}(t-\tau(t))\right\} d t \leq \frac{\left(\overline{r_{1}} b_{2}^{m}+\overline{r_{1}} b_{2}^{M}\right) \omega}{a_{1}^{m} b_{2}^{m}-a_{2}^{M} b_{1}^{M}} .
$$

From formula (8) and formula (13) we get

$$
\int_{0}^{\omega}\left|\dot{X_{1}}(t)\right| d t \leq \frac{2 a_{1}^{M}\left(\overline{r_{1}} b_{2}^{m}+\bar{r}_{1} b_{2}^{M}\right) \omega^{\text {def }}}{a_{1}^{m} b_{2}^{m}-a_{2}^{M} b_{1}^{M}}=W_{1} .
$$

From formula (9) and formula (12) we get

$$
\int_{0}^{\omega}\left|\dot{X_{2}}(t)\right| d t \leq \frac{2 b_{2}^{M}\left(\overline{r_{2}} a_{1}^{m}+\overline{r_{1}} a_{2}^{M}\right) \omega^{\text {def }}}{a_{1}^{m} b_{2}^{m}+a_{2}^{M} b_{1}^{M}}=W_{2} .
$$

Since $\left(x_{1}(t), x_{2}(t)\right)^{T} \in X$, it exists $\theta_{i}, \varphi_{i} \in[0, \omega]$, such that

$$
x_{i}\left(\theta_{i}\right)=\min _{t \in[0, \omega]} x_{i}(t), x_{i}\left(\phi_{i}\right)=\max _{t \in[0, \omega]} x_{i}(t), i=1,2 .
$$

By formula (12), formula (13) and formula (14), we get

$$
\begin{aligned}
& x_{1}\left(\theta_{1}\right) \leq \ln \left[\frac{\left(\overline{r_{1}} b_{2}^{m}+\overline{r_{1}} b_{2}^{M}\right) \omega}{a_{1}^{m} b_{2}^{m}-a_{2}^{M} b_{1}^{M}}\right] \stackrel{\text { def }}{=} V_{11}, \quad x_{1}\left(\varphi_{1}\right) \geq \ln \left(-\frac{\overline{r_{1}} \omega}{a_{1}^{M}}\right) \stackrel{\text { def }}{=} V_{12} \\
& x_{2}\left(\theta_{2}\right) \leq \ln \left[\frac{\left(\overline{r_{2}} a_{1}^{m}+\overline{r_{1}} a_{2}^{M}\right) \omega}{a_{1}^{m} b_{2}^{m}+a_{2}^{M} b_{1}^{M}}\right] \stackrel{\text { def }}{=} V_{21}, \quad x_{1}\left(\varphi_{1}\right) \geq \ln \left(\frac{\overline{r_{2}} \omega}{b_{2}^{M}}\right) \stackrel{\text { def }}{=} V_{22} .
\end{aligned}
$$

So that

$$
x_{i}(t) \leq x_{i}\left(\theta_{i}\right)+\int_{0}^{\omega}\left|\dot{x_{i}}(t)\right| d t \leq V_{i 1}+W_{i}, \quad x_{i}(t) \geq x_{i}\left(\varphi_{i}\right)-\int_{0}^{\omega}\left|\dot{x_{i}}(t)\right| d t \leq V_{i 2}-W_{i} .
$$

It is obvious that $V_{i j}, W_{i}(i, j=1,2)$ has nothing with choose of $\lambda$. Thus the following formula (15) has a unique positive solution $\left(z_{1}^{*}, z_{2}^{*}\right) \in$ int $R_{+}^{2}$.

$$
\left\{\begin{array}{l}
-\overline{r_{1}}-\overline{a_{1}} z_{1}+\overline{b_{1}} z_{2}=0 \\
\overline{r_{2}}+\overline{a_{2}} z_{1}-\overline{b_{2}} z_{2}=0
\end{array}\right.
$$


Let $V=\max \left\{\left|V_{i 1}+C_{i}\right|+V_{1},\left|V_{i 2}+C_{i}\right|+V_{1}, i=1,2\right\}$, where $V_{1}>0$ is sufficiently large and $\left\|\left(\ln z_{1}^{*}, \ln z_{2}^{*}\right)^{T}\right\|=\left|\ln z_{1}^{*}\right|+\left|\ln z_{2}^{*}\right|<V_{1}$. Then, $\nabla=\left\{x(t)=\left(x_{1}(t), x_{2}(t)\right)^{T} \in X:\|x\|<V\right\}$, then $\nabla$ satisfying the first condition for Lemma 1 . When $x \in \partial \nabla \cap \operatorname{KerL}=\partial \nabla \cap R^{2}$,

$$
Q N x=\left(\begin{array}{l}
-\overline{r_{1}}-\overline{a_{1}} z_{1}+\overline{b_{1}} z_{2}=0 \\
\overline{r_{2}}+\overline{a_{2}} z_{1}-\overline{b_{2}} z_{2}=0
\end{array}\right) \neq 0 .
$$

So that $\operatorname{deg}\{J Q N, \nabla \cap \operatorname{KerL}, 0\} \neq 0, \nabla$ satisfying all the conditions for Lemma 1 . We have that there is at least a solution with $L x=N x$ on $D o m L \cap \bar{\nabla}$ from Lemma 1 .

Let $y_{1}^{*}(t)=\exp \left\{x_{1}^{*}(t)\right\}, \quad y_{2}^{*}(t)=\exp \left\{x_{2}^{*}(t)\right\}$, when $a_{1}^{m} b_{2}^{m}>a_{1}^{M} b_{2}^{M}, \quad\left(y_{1}^{*}(t), y_{2}^{*}(t)\right)^{T}$ is positive periodic solution for system (1) which the length of $\omega$. Hence, there is at least a positive periodic solution for system (1).

Theorem If $a_{1}^{m} b_{2}^{m}>a_{1}^{M} b_{2}^{M}$, then $\left(y_{1}^{*}(t), y_{2}^{*}(t)\right)^{T}$ is a positive periodic solution for system (1). In other word, there is at least one positive periodic solution for system (1).

\section{Acknowledgements}

This research was financially supported by the National Science Foundation of Zhejiang Province (LY12A01010) and by the College Students' Scientific and Technological Innovation of Zhejiang Province (2015R411035).

\section{References}

[1] Wang, G.X., et al. (2006) Ordinary Differential Equation. Higher Education Press, Beijing. (In Chinese)

[2] Lin, Z.S. (2006) Population Dynamics. Science Press, Beijing. (In Chinese)

[3] Lin, S.Q. and Lu, Z.Y. (2006) Permanence for Two-Spcies Lotka-Volterra System with Delays. Mathematical Biosciences and Engineering, 3, 137-144.

[4] Jiang, Q.Y., Xie, J.X. and Ye, J. (2003) Mathematical Model. Higher Education Press, Beijing. (In Chinese)

[5] Xie, X.D. and Chen, F.D. (2014) Study on the Dynamics of Cooperative Population Model. Science Press, Beijing. (In Chinese)

[6] Chen, L.S. and Liu, S.Q. (2010) Study on Population Biology Model of Stage Structure. Science Press, Beijing. (In Chinese)

[7] Chen, L.S. (1988) Essential Mathematical Biology. Science Press, Beijing.

\section{Submit or recommend next manuscript to SCIRP and we will provide best service for you:}

Accepting pre-submission inquiries through Email, Facebook, LinkedIn, Twitter, etc.

A wide selection of journals (inclusive of 9 subjects, more than 200 journals)

Providing 24-hour high-quality service

User-friendly online submission system

Fair and swift peer-review system

Efficient typesetting and proofreading procedure

Display of the result of downloads and visits, as well as the number of cited articles

Maximum dissemination of your research work

Submit your manuscript at: http://papersubmission.scirp.org/ 